\title{
Entwicklungspotenzial im Kunstunterricht sowie in der Lehrer*innenausbildung
}

\author{
Kreativitätsförderung von ungarischen Hochschulstudierenden \\ Heidelinde Balzarek ${ }^{1}$, Katinka Szettele ${ }^{2}$
}

https://doi.org/10.53349/resource.2021.i16.a996

\section{Zusammenfassung}

In der vorliegenden Studie wird auf das Thema Förderung des kreativen Potenzials im schulischen Kontext eingegangen. Es wird über den Einsatz kreativitätsfördernder Methoden sowie Einstellungen von Lehrpersonen gegenüber Kreativität anhand jüngster Forschungsergebnisse diskutiert. Im weiteren Teil der Studie wird auf den Kreativitätsbegriff des Lehrplans bzw. im fachdidaktischen Diskurs reflektiert und die Relation von Kunst und Kreativität als eine Resonanzbeziehung dargestellt. Das Entwicklungspotenzial im Kunstunterricht wird am Beispiel einer Online-Werkstattarbeit präsentiert, die in einer partnerschaftlichen Kooperation zweier Ausbildungsinstitutionen (Pädagogische Hochschule Niederösterreich und Eötvös-József-Hochschule Baja, Ungarn) im Studienjahr 2020/2021 verwirklicht wurde. Zum Abschluss werden sowohl die pädagogischpraktischen Ansätze zur Kreativitätsförderung von Hochschulstudierenden in Ungarn als auch Ergebnisse der mit dem Instrument TSD-Z durchgeführten Untersuchung vorgelegt.

Keywords:

Kreativität

Kunst

Pädagogik

Lehrer*innenausbildung

\section{Einleitung}

Die Entwicklung des kreativen Potenzials ist ein gängiges Thema in der internationalen Forschung und wird auch in der Bildungspolitik mehrerer Länder hervorgehoben. Über ihr Wesen und ihre praktische Umsetzung wird jedoch weniger gesagt, obwohl eine Förderung ohne entsprechendes schulisches Umfeld nicht existieren kann. Die Bedeutung der Lernumgebung wird von Beghetto und Kaufmann (2014) als einer der wichtigsten Faktoren hervorgehoben, von denen die Entwicklung des kreativen Potenzials abhängt. Im Folgenden werden Merkmale des kreativitätsfördernden Unterrichtsklimas dargestellt, die laut internationalen Forschungen das effektive und erfolgreiche Lernen begünstigen. Darüber hinaus wird die Frage aufgeworfen, wie sich Lehrpersonen dem Thema Kreativität annähern, welche Ansichten sie haben und was für Missverständnisse bzw. implizite Überzeugungen im Zusammenhang mit dieser Thematik typisch sind.

\footnotetext{
1 Pädagogische Hochschule Niederösterreich, Mühlgasse 67, 2500 Baden.

E-Mail: heidelinde.balzarek@ph-noe.ac.at

2 Eötvös József Főiskola, Szegedi út 2, 6500 Baja, Ungarn

E-Mail: szettelekatinka@gmail.com
} 


\section{Kreativität im schulischen Kontext}

\subsection{Lernen in einem kreativen Umfeld - Wege der Kreativitätsförderung}

Internationale Literatur zur Entwicklung von Kreativität im pädagogischen Kontext findet sich unter anderem bei Beghetto und Kaufmann (2014). Die Autoren weisen darauf hin, dass im Vier-C-Modell der MehrebenenKreativität vor allem das mini-c und das Little-c für die Pädagogik relevant sind, die die niedrigeren Stufen der Kreativität, die sogenannte Alltags-Kreativität, repräsentieren. Laut ihrem Konzept ist die frühe Lebensphase durch das mini-c gekennzeichnet, das sich im freudigen Spiel der Kinder, im Erforschen der Welt, im spontanen Wissenserwerb beobachten lässt. Die ersten Manifestationen von mini-c finden sich vor allem bei Kindern im Grundschulalter und äußern sich in einer Vielzahl von kreativen Aktivitäten, die bereits mit einem bestimmten Bereich verbunden sind. Positives Feedback ist entscheidend für das Erreichen höherer Ebenen des kreativen Ausdrucks, der ein Individuum nach ausreichender Übung erstens zum Little-c, dann zur Experten-Ebene (ProC) bzw. zum Big-C mit künstlerischen oder wissenschaftlichen Spitzenleistungen führen kann.

Das Paradigma des kreativen Lehrens und Lernens kann im Zusammenhang mit einem effektiven und effizienten Wissenserwerb verstanden werden. In der Tat, wenn die Kreativität der Lernenden anerkannt und darauf aufgebaut wird, steigt die akademische Leistung der Lernenden (Fisher \& Williams, Hrsg., 2004). Beghetto und Kaufman (2014) schlagen die Integration von Kreativität in die allägliche pädagogische Praxis („classroom creativity“) vor und argumentieren, dass geeignete Methoden und Techniken als Kreativitätsstimulatoren im Lernprozess wirken können.

Bereczki (2018) diskutiert in ihrer Meta-Analyse ausführlich über die Wirksamkeit der Methoden zur Kreativitätsförderung. Die Studien zeigen, dass Kreativität sowohl in jüngeren als auch in älteren Jahren entwickelt werden kann und der Einsatz kreativitätsfördernder Methoden für eine breite Palette von Lerngruppen effektiv ist (Ma, 2006; Scott, Leritz \& Mumford, 2004a, 2004b; Lai, Yabro, DiCerbo \& DeGeest, 2018). Die erfolgreichste Art und Weise der Entwicklung sind vor allem Interventionen, die sich auf die Denkfähigkeit konzentrierten und in einer spezifischen Disziplin praxisorientiert eingesetzt wurden (Scott et al., 2004b). Dabei wird das divergente Denken besonders bevorzugt, das als Grundlage zahlreicher Techniken zur Kreativitätsförderung dient (siehe Brainstorming, Mind Map, Synectics-Methode, KJ-Methode etc.)

Klaus K. Urban gibt 25 Anregungen an, damit sich das Kreativitätspotenzial der Schüler*innen entfalten kann. ${ }^{1}$ Er schlägt vor, dass die Lehrpersonen freies Spiel unterstützen bzw. Fragen stellen sollten, die freies, gleichwohl sachbezogenes Phantasieren ermöglichen und die Neugier erwecken. Empfohlene Techniken sind: nach Analogien und Metaphern suchen (z. B. "Was wäre wenn?"), etwas ins Gegenteil verkehren, Ideen, Sachverhalte, Gegebenheiten systematisch befragen, untersuchen, analysieren, verändern, reformulieren, neu kombinieren etc. Lehrpersonen sollten selbst-initiierte Fragen unterstützen und Humor wertschätzen, denn so hat man gleichzeitig Abstand und Nähe zu einem Gegenstand mit mehrperspektivischer Betrachtung. Im Rahmen einer kreativen Pädagogik sollte man die Kinder sensibel für Stimuli aus der Umwelt machen bzw. ihre Toleranz und Akzeptanz für neue, möglicherweise überraschende Gedanken entwickeln. Teil der Erziehung sind das Zulassen von Fehlern und Irrwegen und zugleich das Verhindern von Sanktionen von Seiten der Kamerad*innen. Lehrpersonen sollten Situationen, die schöpferisches Handeln anregen oder erfordern, schaffen bzw. provozieren und für einen angemessenen Wechsel von aktiven und ruhigen, Muße ermöglichenden Perioden sorgen. Man sollte die Freude am Experimentieren fördern und eine volle Ausarbeitung bzw. Verwirklichung von Ideen unterstützen.

\subsection{Einstellungen von Lehrpersonen gegenüber Kreativität}

Obwohl bildungspolitische Richtlinien und Lehrplanvorgaben die Förderung der Kreativität seit Jahrzehnten unterstützen, reichen sie nicht aus, wenn es um die Umsetzung in der Alltagspraxis geht. Denn die Ansichten der Lehrkräfte über Kreativität bestimmen gleichermaßen die Wirksamkeit der Kreativitätsentwicklung. Internationale Forschungen (Aljughaiman \& Mowrer-Reynolds, 2005; Bereczki \& Kárpáti, 2018) zeigen, dass Lehrpersonen die Entwicklung von Kreativität zwar für wichtig halten, sie diese aber meist nur außerhalb des Klassenzimmers (getrennt vom akademischen Lernen) als angemessen erachten. Die Förderung der Kreativität wird eher als fakultative Ergänzung zu ihrer sonstigen Tätigkeit angesehen. Demnach gilt Kreativitätsförderung im Unterricht als Zeitverschwendung, aber als fakultative Tätigkeit kann sie auch leicht abgelehnt werden, denn sie sollte extra Zeit und Energie in Anspruch nehmen. 
Ein weiteres Problem ist, dass implizite Theorien der Lehrpersonen gegenüber Kreativität in vielen Fällen einen negativen Einfluss auf die Entfaltung kreativer Fähigkeiten von Schüler*innen haben können (Plucker et al., 2004; Bereczki, 2016). Laut der Studie von Bereczki und Kárpáti (2018), die Ansichten von Lehrer*innen über Kreativität anhand von 53 internationalen Studien analysiert, gibt es beispielsweise eine weit verbreitete Vorstellung, dass Kreativität einfach nur Spaß macht und keine Anstrengung oder Vorkenntnisse erfordert, sodass sie mit dem eher als intellektuell angesehenen Prozess des Lernens unvereinbar ist. Das ist deswegen problematisch, weil es in diesem Fall keine Rechtfertigung für eine Entwicklungsarbeit im Klassenzimmer gibt. In diesem Fall nutzen die Lehrkräfte die Angelegenheit der sog. "kreativen Mikromomente im Klassenzimmer" (Beghetto, 2013) nicht aus, die in einem kreativen Lehr-Lern-Prozess Anlass zur Kreativitätsförderung bietet (z. B. eine unerwartete Frage, Antwort oder Idee einer Schülerin*eines Schülers, die zur weiteren Diskussion führen kann). Solche negativen Überzeugungen (die naturgemäß auch unbewusst existieren können) können Förderungsmaßnahmen hemmen oder stören (Theurer, 2015).

Eine weitere Barriere für die Entwicklung von Kreativität kann die Überzeugung sein, dass sie eine angeborene Fähigkeit wäre, gleichzusetzen mit Naturtalent oder Intelligenz, und dass ihre Entwicklung nur für die Künste relevant ist. Laut der Studie von Bereczki und Kárpáti (2018) halten Lehrer*innen Kreativität für wichtig, aber viele von ihnen assoziieren sie nur mit den Künsten und beschränken ihre Entwicklung auf visuelle Bildung, Theater oder Musik. Dagegen spricht, dass sich in jüngster Zeit gezeigt hat, dass „Kreativität integraler Bestandteil von einem breiten Spektrum an Wissen und Fähigkeiten ist, einschließlich wissenschaftlichem Denken, Entrepreneurship, Design Thinking und Mathematik" (Fadel et al., 2017, S. 129).

Es gibt noch einen Widerspruch, wenn man Lehrpersonen befragt. Nämlich, dass sie gerne mit kreativen Schüler*innen arbeiten und kreatives Verhalten im Unterricht wertschätzen, jedoch mit Kreativität eher negative Persönlichkeitseigenschaften assoziieren (Westby \& Dawson, 1995). Noch in der frühen Untersuchung von Torrance (1965) zeigt sich, dass Lehrpersonen in den fünf untersuchten Nationen "dazu tendieren, solche Schüler abzulehnen, die Fragen stellen, im Unterricht Vermutungen äußern, in ihren Gedanken und Urteilen von anderen unabhängig sind, intuitiv handeln und risikobereit sind oder Autoritäten infrage stellen" - so Theurer (2015). Darüber hinaus fallen kreative Schüler oft störend auf (Scott, 1999), und schließlich argumentieren Lehrpersonen, die Kreativität im Klassenzimmer ablehnen, dass Kreativität das Brechen von Regeln mit sich bringt, die Autorität untergräbt und zu Konfrontationen führt (Grigorenko, 2019).

All dies deutet darauf hin, dass, obwohl die Bildungspolitik des letzten Jahrzehnts von einem Streben nach innovativen Veränderungen dominiert wurde, die Ansichten der Lehrer*innen über Kreativität einen großen, wenn nicht größeren Einfluss auf die Umsetzung der Prinzipien haben. Diese bilden die Grundlage der täglichen pädagogischen Praxis, die die Entwicklung des kreativen Potenzials der Lernenden bestimmt. Wichtig ist dabei, dass die Förderung der Kreativität nicht nur positive Einstellungen, sondern auch entsprechende implizite Überzeugungen von der Lehrkraft erfordert sowie eine gewisse Flexibilität, die nicht unbedingt durch den Lehrplan unterstützt wird.

Laut der Studie von Theurer (2015) wird in der internationalen Forschung oft argumentiert, dass die Einstellungen der Lehrpersonen zu Kreativität und zu kreativitätsförderndem Unterrichten erhoben und zum tatsächlichen unterrichtlichen Handeln in Beziehung gesetzt werden sollten. Die Forscher (Westby \& Dawson, 1995; Aljughaiman \& Mowrer-Reynolds, 2005; Morgan \& Forster, 1999; Moran, 2010; Sawyer, 2012) sind der Überzeugung, dass eine entsprechende Ausbildung von Lehrkräften notwendig wäre, um Unsicherheiten oder Widerstände abzubauen und Methoden schulischer Kreativitätsförderung bekannt zu geben.

\section{Kunstunterricht - Einsatz und Umgang mit dem Kreativitätsbegriff}

Der deutsche Kunstpädagoge Rudolf Seitz sieht im Kunstunterricht eine Kreativitätserziehung, in deren Fokus primär die Persönlichkeitsentwicklung steht. Als ein weiteres Ziel des Kunstunterrichts legt er die Ermöglichung subjektiver Kreativität fest. Er stellt in seinen Reflexionen auch die Frage, ob eine Gesellschaft überhaupt kreative Kinder möchte. Denn das deklarierte Ziel des Kunstunterrichts stellt die Entwicklung kreativer Persönlichkeiten dar. Diese müssen mit einer sich radikal verändernden Welt umgehen und darin bestehen können. Seitz fixiert den Kreativitätsbegriff als ein grundlegendes Bildungsthema (Seitz, 2009). Der Begriff dominiert seine Aussagen und sein Handeln als Kunstpädagoge und Ausbildner.

Im aktuellen österreichischen Lehrplan der Grundschule für das Fach Bildnerische Erziehung soll folgendes Zitat die Funktion und die Aufgaben des Kunstunterrichts beschreiben:

In der bildnerischen Tätigkeit geht es einerseits um das Kennenlernen, Erproben und Anwenden von Ausdrucksmöglichkeiten in Bereichen wie Grafik, Malerei, Plastik, Raum, Schrift, Fotografie, Film, 
Video, Neue Medien, Spiel und Aktion sowie Gestaltung der eigenen Umwelt. Andererseits geht es um die Entwicklung des bildhaften Denkens und persönlichkeitsbezogener Eigenschaften wie Offenheit, Flexibilität, Experimentierfreude, Einfallsreichtum, Sensibilität, Konzentrationsfähigkeit, Ausdauer, Kooperationsbereitschaft und Rücksichtnahme (Lehrplan der Volksschule, S. 173).

Das Fach weist die zwei Teilbereiche „Bildnerisches Gestalten“ sowie „Wahrnehmen und Reflektieren“ auf, die die sachliche und stoffliche Struktur des Unterrichtsgegenstandes sowie die mit inm verbundenen Lernchancen darstellen. Die Teilbereiche beinhalten: Grafik, Malerei, Plastik/Objekt/Raum, Fotografie und Film/Video, Spiel und Aktion. Die beiden Teilbereiche sind in der Praxis in eine enge wechselseitige Beziehung zu bringen.

Die Anerkennung der individuellen Äußerungen der Kinder steht im Fokus. Insgesamt soll die gesamte unterrichtliche Tätigkeit von einer positiven Erwartungshaltung getragen sein. Die positive Verstärkung steht bei Wertungen im Vordergrund. Manchmal kann auch die Umsetzung einer Technik der Ausgangspunkt sein, Technik und Darstellungsabsicht sollen aber in Korrelation stehen. Fantasie und der bildnerische Einfallsreichtum sollen geweckt werden. Jedes Kind soll orientiert sein, aber gleichzeitig so viel Spielraum haben, dass es seine persönlichen Ideen und Vorlieben einbringen und sich mit dem fertigen Werk identifizieren kann. Dieser Gestaltungsspielraum ist auch wichtig, um den heterogenen Entwicklungsphasen und Begabungen innerhalb einer Klasse gerecht zu werden. Im Vergleich mit den anderen kann jedes Kind sich in seiner Eigenart erkennen und gleichzeitig die Eigenart der anderen anerkennen. Im Lehrplan wird mit dem Kreativitätsbegriff sehr sparsam umgegangen. Nur im Zusammenhang mit schematischem Festlegen von Gestaltungsweisen und dem bloßen Ausmalen von Vorlagen wird festgestellt, dass dies zu unterlassen ist, weil dadurch die kreativen Fähigkeiten der Kinder unterbunden werden. ${ }^{2}$

Ein ebenso zurückhaltender Einsatz des Kreativitätsbegriffs lässt sich im „SCHÜLER\&INNEN_KOMPETENZEN_BE LEITFADEN10|2013“ feststellen. Dieser Text wurde von der Arbeitsgruppe "SchülerInnenkompetenzen in Bildnerischer Erziehung" entwickelt und erstellt. Der Kompetenzbegriff und der Bildbegriff werden im fachlichen Diskurs fassbar gemacht und alle Schularten dahingehend beschrieben. Der Begriff Kreativität kommt nur ein einziges Mal in abgewandelter Form vor. Im Bereich Bilder erfinden wird folgende über alle Schultypen gespannte Kompetenz gelegt: Kreativstrategien anwenden (Assoziationsfreude, Perspektivenwechsel, ...). ${ }^{3}$

In Carl-Peter Buschkühles Werk „Künstlerische Bildung. Theorie und Praxis einer künstlerischen Kunstpädagogik“ (2017) findet der Kreativitätsbegriff keine bemerkenswerte Erwähnung, während dieser Terminus in Veröffentlichungen von Constanze Kirchner intensiv zum Einsatz kommt. Das erste Wort der Einleitung ihres Buches „Kunst - Didaktik für die Grundschule“ (2013) ist Kreativität. Nicole Berner hat in diesem Zusammenhang mit dem Sammelband „Kreativität im kunstpädagogischen Diskurs" (2018) einen wichtigen Beitrag geleistet, den Begriff Kreativität wieder verstärkt in der Theoriebildung zu berücksichtigen. Sie betont im Vorwort, wie wichtig ein reflektierter Umgang mit Kreativität ist, denn auch ethische und gesellschaftliche Funktionen sind mit diesem Bildungsziel verbunden.

Dieser knappe Überblick, wie der Kreativitätsbegriff im Diskurs der Kunstdidaktik eingesetzt wurde und wird, zeigt das wissenschaftliche Spannungsfeld auf.

\subsection{Kunst und Kreativität - eine Resonanzbeziehung}

Nach Schuster (1997) kann die Bildende Kunst als ein kreatives Feld betrachtet werden, in dem sich Kreativität im Sinne einer künstlerischen Kreativität manifestiert. Kunst darf nicht nur als kunsthistorische Größe betrachtet werden, sondern auch als ein sich ständig transformierendes, zeitgeistiges Phänomen (Ursprung, 2012). Die rezeptive Auseinandersetzung mit Kunstwerken fordert Schüler*innen, den Sinn, d. h. dessen Bedeutung, zu erschließen (Kirchner \& Peez, 2009), und bietet viele Möglichkeiten für kreatives Verhalten. Die Grenzen der Disziplinen werden dabei überwunden und Brücken zu anderen künstlerischen Ausdrucksweisen geschlagen, wie zur Musik, Literatur und zu theatralen Kunstformen. Ebenso finden Annäherungen zu Technik, Naturwissenschaft und Mathematik statt. Kunst wird über Sinne wahrgenommen. Dieses Erleben und Erfahren ermöglicht individuelle Erkenntnisprozesse, die von pädagogischer Relevanz sind. Kunst kommuniziert auf diverse Art und Weise durch eine eigene Formen- und Bildsprache, die als Ausdruck des herrschenden Zeitgeistes von den Kunstschaffenden kreiert wurde. In diesen künstlerischen Prozessen manifestiert sich ein kreatives Potential, das aber auch in der Kunstrezeption festzumachen ist.

Kunst und Kreativität bedingen einander. Buschkühle (2017) sieht in Kunstwerken die Verbindung von Geist und Materie, eine Vergeistigung des Materials im Ausdruck, eine Materialisierung der Vorstellung im Bild. 
Er bringt Kunst und Philosophie auf eine Ebene und bezeichnet Kunst als Philosophie mit anderen Mitteln. Die Kunst bildet die Basis für künstlerische Bildung. Künstlerische Prozesse initiieren bei Kindern und Jugendlichen künstlerisches, unabhängiges und kreatives Denken. Dadurch können sie an Kunst und Kultur teilhaben (Buschkühle, 2017).

Ebenso findet die Ästhetische Bildung ihren Bezugspunkt in der Kunst, indem durch Auslegungsprozesse (Otto \& Otto, 1987), praktisch-rezeptive Methoden (Krautz, 2005) sowie durch partizipative Methoden eigene Entwürfe von Wirklichkeit ermöglicht und damit Wege zur Auseinandersetzung mit Kunst eröffnet werden.

In diesem Zusammenhang muss der Resonanzbegriff fokussiert werden, den der Soziologe Hartmut Rosa als eine Metapher für Beziehungsqualitäten einführt. Durch den Resonanzbegriff entfaltet sich ein enormes Anregungspotential für die Untersuchung von Weltverhältnissen in fast allen Bereichen des menschlichen Lebens. Er bietet keine ausgearbeitete Philosophie oder Soziologie der Kunst an, aber er dekliniert sämtliche Kunstformen und deren Ausdruck mit konkreten Beispielen durch, wobei er auch in diesem Zusammenhang die Begriffe "Glück" und "Schönheit" aufgreift. Ästhetische Resonanz wird durch seine Darstellung zu einem Experimentierfeld für die Anverwandlung unterschiedlicher Muster der Weltbeziehung, also der kreativen Gestaltungsprozesse.

Einen Hauptantriebsmotor für künstlerische Produktion und Rezeption und damit für die Entfaltung von Kreativität sieht Hartmut Rosa in der Sehnsucht nach einer resonanten Weltbeziehung. Oft wendet sich die*der Kunstschaffende an die Natur und entwickelt durch ihre*seine Kreativität eine Weltbeziehung. Diese kreative Auseinandersetzung manifestiert sich durch Kunstwerke und gestaltet somit den Resonanzraum. Das aktive künstlerische, kreative Tätigsein lässt Resonanzen erfahrbar und spürbar werden. Die Kraft der Kunst bildet eine Sphäre für die Modulation und Exploration von Weltbeziehung, wobei hier die Selbstwirksamkeitserfahrung eine große Rolle spielt. Kunstproduktion setzt er mit einem Resonanzgeschehen gleich, was aber ebenso in der Kunstrezeption geschehen kann, aber nicht muss. Kreativität ist aus dieser Perspektive ein Resonanzgeschehen (Rosa, 2017).

\subsection{Entwicklungspotenzial im Kunstunterricht als Resonanzgeschehen}

Die Lernkultur ist ausschlaggebend, dass sich Kreativität als Potential entwickeln kann. Die Grundlage jeder Kreativitätsförderung ist das Erkennen des kreativen Potentials der Schüler*innen (Berner, 2013; Kirchner \& Peez, 2009; Wichelhaus, 2006). Die Kompetenz, Kreativität zu erkennen, erfordert zum einen ein explizites Verständnis von Kreativität und zum andern ein kritisches Hinterfragen der eigenen, subjektiven Wahrnehmung. Nur wer selbst kreativ ist, kann die Kreativität anderer wahrnehmen und erkennen. Nach Heinelt (1978) können nur kreative Lehrpersonen Schüler*innen in ihrer Kreativität unterstützen und fördern.

In der Kunstpädagogik kommt der Förderung der Kreativität vorrangige Bedeutung zu. Hemmende Faktoren (u. a. Zeitdruck, Leistungsdruck) sollten reduziert werden und dafür geeignete Maßnahmen eingeleitet werden, die differenziert auf die unterschiedlichen Dimensionen der Kreativität abzielen (u. a. Kirchner, 2013; Brügel, 1987; Dinkelmann, 2008; Urban, 2004) Von immenser Relevanz ist das Wissen um Kreativität, das zum fachdidaktischen Grundlagenwissen einer Lehrperson zählt. Gezielte Fördermaßnahmen und ein anregendes, vertrauensvolles Lernklima sind für Kreativitätsentfaltung wichtig (Preiser/Buchholz, 2008). Ebenso sind emotionale und soziale Faktoren ausschlaggebend dafür, wie gelernt und gestaltet wird. Freie, problemlösungsorientierte, kreative Lernprozesse können Unsicherheiten auslösen, denn es gibt unterschiedliche Ergebnisse und oftmals kein eindeutiges Richtig oder Falsch. Die „Kanalisierung des Lernens in der Schule bremst das Explorationsverhalten“ (Einsiedler, 1979, S. 157). Offene Lernformen und Aufgaben in einem geschützten Raum, können die Lernenden ermutigen, sich zu erproben und experimentell mit Situationen umzugehen (Berner, Hess \& Lipowsky, 2018; Chin, 2013; Schäfer, 2006).

\subsection{Die Rolle des ästhetisch-künstlerischen Forschens in der Kreativitätsförderung}

Im Fokus dieses methodischen Ansatzes des künstlerisch-ästhetischen Forschens nach Helga Kämpf-Jansen (2012) steht der Prozess, also die kreative Auseinandersetzung. „Ästhetik“ wird nicht mit der philosophischen Kategorie des „Schönen“ in Verbindung gesetzt, sondern bezieht sich auf die ursprüngliche Bedeutung, nämlich sinnliche Wahrnehmung. Diese Forschung, ausgehend von unterschiedlichen Themenfeldern, soll „ästhetische Verhaltensweisen, Handlungs- und Erkenntnisformen" ermöglichen. Damit bietet sich ein kreatives Experimentieren, also ein vielfältiger, handlungsorientierter Umgang mit unterschiedlichsten Dingen an, wie 
z. B. sammeln, ordnen, dekorieren, arrangieren, dokumentieren, präsentieren. Grenzen von Kunst, Musik, Literatur und Film werden ausgelotet und es entstehen intermediale Produkte, welche die Perspektive über die Fachgrenzen in andere wissenschaftliche Bereiche erweitern.

Genauso kann von Inhalten und Themen ausgegangen werden, mit denen die Lernenden in Resonanz gehen und welche sie in künstlerisch ästhetischer Form umsetzen. Fragen nach Bedeutung und vertiefender Betrachtung werden aufgeworfen, unterschiedliche Thematiken beleuchtet und im Prozess bearbeitet. Wie sich eine Fragestellung weiterentwickelt, ist von den Forschungsansätzen und deren Fortschritten abhängig. Jeder Arbeitsprozess verläuft individuell, manchmal fließend, dann wieder mit zahlreichen Brüchen. Die Konstitution und Orientierung des Individuums im Hier und Jetzt sind Bestandteil der Auseinandersetzung, wie auch zeitgeistige Fragen in diesem Zusammenhang, wie die nach Erinnern und Vergessen und nach ästhetischem Denken.

Helga Kämpf Jansen (2012) stellt mit ihren fünfzehn Thesen zum ästhetischen Forschen viele Ansätze zum kreativen Vorgehen zur Diskussion. Es sind keine Dogmen, sondern Impulse und Anregungen. Diese Methode lässt sich ausgezeichnet mit der agilen Hochschuldidaktik nach Christof Arn (2020) kombinieren. Diese Präsenzdidaktik, Co-Didaktik bzw. Didaktik als Performance zielt auf agiles Lernen ab, wobei agile Werte, Prinzipien und Methoden fokussiert werden. Selbstorganisation und Eigenverantwortung stehen beim lernzentrierten, handlungskompetenzorientierten und mediengestützten Lernen und Forschen im Mittelpunkt. Die Lehrenden übernehmen die Rolle des Lernbegleitens oder Unterstützens. Auf diese Art und Weise können kreative Lern- und Arbeitsprozesse initiiert und begleitet werden (Christof Arn, 2020).

\section{Werkstattarbeit mit Hochschulstudierenden}

Die Erasmus-Partnerschaft für Zusammenarbeit der zwei Ausbildungsinstitutionen (Pädagogische Hochschule Niederösterreich und Eötvös-József-Hochschule Baja, Ungarn) kann auf eine Vergangenheit von etwa 15 Jahren zurückblicken. Im Studienjahr 2020/2021 wurde das Modul „Bildnerisches Gestalten“ den Hochschulstudierenden online angeboten und in Kooperation von zwei Kolleginnen (Autorinnen des Artikels) mit dem Schwerpunkt Kreativität gemeinsam veranstaltet. Unser Ziel war, inspiriert von der Idee unterschiedlicher Aus- und Weiterbildungsprogramme im Bereich der Kreativitätspädagogik, dass Lehramtsstudierende (sowie Lehrpersonen, die bereits im Berufsleben stehen) die eigenen kreativen Potentiale im Rahmen der Workshoparbeit erkennen, nutzen und entwickeln. Bei diesem kreativen Akt spielten die Ideenfindung sowie selbstständiges Gestalten eine besondere Rolle. Außerdem hielten wir es für wichtig, die Reflexion, eine persönliche Stellungnahme bzw. Beziehung zu Kunstwerken zu ermöglichen (vgl. Resonanzgeschehen im Kunstunterricht).

Nach einer kurzen Frage-und-Antwort-Runde, die als Warm-up-Spiel eingesetzt wurde, wurden zuerst Zitate zum Thema Kreativität gezeigt. Mithilfe unterschiedlicher Definitionen der Kreativität wie z. B. „Kreativität ist Intelligenz, die Spaß hat" von Albert Einstein oder „Wahre Kreativität entsteht immer aus dem Mangel" von Wolfgang Joop wurden die Assoziationen der Studierenden geweckt und sie konnten auch Kommentare hinzufügen sowie ihre Meinungen zum Kreativitätsbegriff äußern. Bei dem Gespräch über kreative Aktivitäten wurden Bilder gezeigt und die Teilnehmer*innen versuchten, eine Reihenfolge festzustellen sowie sich persönlich einzubringen. Sie wurden aufgefordert, sich in Kleingruppen (in Teams auch durch Erstellung von Gruppenräumen möglich) zu äußern, welche Aktivitäten sie auch machen oder gerne machen würden. Es wurde ebenso die Frage gestellt, wann sie letztes Mal künstlerisch gearbeitet hätten. Zuzüglich konnten sie Gegenstände zeigen, die sie selbst kreiert hatten. Bei diesem Punkt wurde ihnen die bereits dargelegte Theorie der Mehrebenen-Kreativität vorgestellt und das Vier-C-Modell von Beghetto und Kaufmann (2014) präsentiert.

Als Einstieg zu einer gestalterischen Werkstattarbeit wurde mit den Studierenden ein Kreativitätstest online durchgeführt. Sie sollten nämlich den in Teams hochgeladenen TSD-Z (A) (Test zum schöpferischen Denken - Zeichnerisch) von Urban \& Jellen (1995) herunterladen und ausdrucken. Das Blatt beinhaltet sechs figurale Elemente (Halbkreis, Punkt, große Ecke, Schlangenlinie, Strichlinie, kleines liegendes U außerhalb des Rahmens), die zum Zeichnen bzw. Weiterzeichnen anregen. „Der TSD-Z versteht sich als Screening-Instrument, als ein Verfahren zur Grobeinschätzung von kreativen Teilkomponenten des schöpferischen Handelns eines Menschen" (Harecker, 2000, S. 52-53). Der Test vertritt eine Herangehensweise (im Gegensatz zu den eingeschränkten Konzepten der herkömmlichen Kreativitätstests, die lediglich quantitative Kriterien des divergenten Denkens berücksichtigen), die das Gestalthafte eines kreativen Produkts zu erfassen versucht (Urban, 2011). Dazu dienen die 14 Auswertungskriterien, die auf folgende Aspekte fokussieren: 
- Weiterführung der figuralen Fragmente

- Zusätzliche Ergänzungen, Finden und Einbinden neuer Elemente

- Herstellung zeichnerischer und/oder thematischer Verbindungen (Komposition)

- Begrenzungsüberschreitung: Die Überschreitung einer scheinbar vorgegebenen Begrenzung

- Verwendung von Perspektive

- $\quad$ Ausdruck von Humor oder Affektivität

- $\quad$ Fiktionale und/oder abstrakte Zeichnungen

- $\quad$ Verwendung von Zeichen und Symbolen

- Unkonventionalität: nicht stereotypische Verwendung der vorgegebenen Fragmente

Die Studierenden wurden darum gebeten, die Testblätter herzunehmen und sie innerhalb von 15 Minuten zu ergänzen. Die Anweisung zur zeichnerischen Produktion lautet folgendermaßen: Jemand fing zu zeichnen an und hörte auf, bevor er wusste, was es überhaupt hätte werden sollen; nun soll die Zeichnung zu Ende gemacht werden, wie immer man will - man kann nichts falsch machen.

Die kreativen Merkmale der Zeichnungen wurden anhand der oben dargelegten Kriterien mit den Teilnehmer*innen besprochen. Es wurden Fragen gestellt wie „Wer hat die Elemente miteinander verknüpft?, Wer hat auch außerhalb des Rahmens gezeichnet?" oder "Wer hat die Schlangenlinie nicht als Schlange beendet bzw. aus der großen Ecke kein Haus gemacht?". Die Studierenden zeigten ihre Werke am Bildschirm.

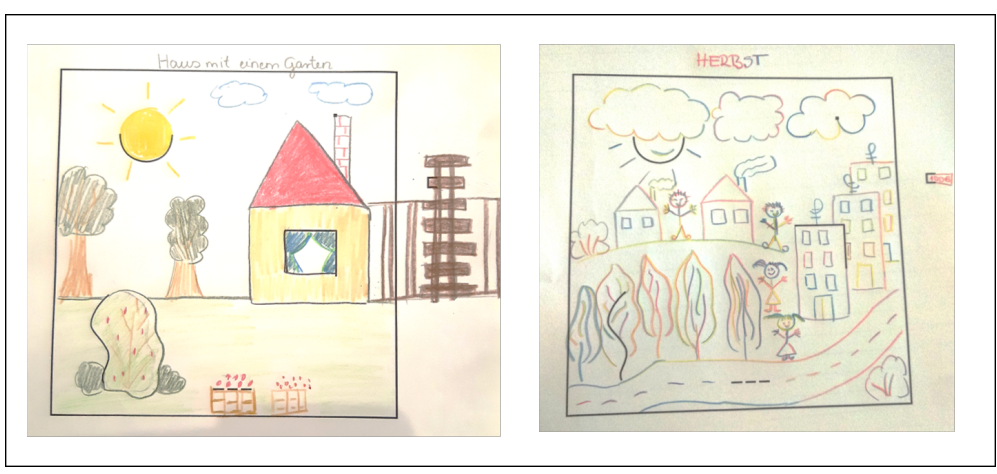

Bild 1: Erstellte TSD-Z (A) Testblätter von ungarischen Hochschulstudierenden

Durch die Besprechung des Kreativitätstests wurden die kreativen Komponenten einer Komposition bewusst gemacht und die Studierenden konnten das eigene kreative Potenzial einschätzen sowie ihre eigene kreative Gestaltungsweise kennen lernen. Diese Selbsterfahrung befähigt die Studierenden (bereits Lehrpersonen), den Test auch in der eigenen Unterrichtspraxis erfolgreich einzusetzen.

Aufgrund der Ergebnisse können Fördermaßnahmen für Gruppen oder Einzelne ergriffen werden, und zwar sowohl für schwächere Schüler als auch für solche mit besonderen kreativen Potentialen. (...) Dazu müssten Lehrkräfte sich auch fragen, ob und inwiefern ihr eigenes Verhalten, ihr Unterrichtsund Lehrstil eher zur Hemmung und Verhinderung als zur Anregung, Stimulierung und Förderung kreativer Verhaltensweisen ihrer Schülerinnen und Schüler beiträgt. (Urban, 2011, S. 21)

Der TSD-Z kann generell in der Schule schon ab der ersten Klasse eingesetzt werden. Laut Urban (2011) können Lehrer*innen dadurch ihre Beobachtungen, Eindrücke und Rückschlüsse objektivieren, ergänzen und gegebenenfalls korrigieren.

\begin{tabular}{|c|c|c|}
\hline Testwerte & Erreichte Punktzahl in \% & Klassifikation (Form A) \\
\hline 29 & 40 & C \\
\hline 36 & 50 & C \\
\hline 33 & 45 & C \\
\hline 37 & 51 & D \\
\hline 15 & 20 & A \\
\hline
\end{tabular}




\begin{tabular}{|c|c|c|}
\hline 35 & 48 & $\mathrm{C}$ \\
\hline 42 & 58 & $\mathrm{D}$ \\
\hline 39 & 54 & $\mathrm{D}$ \\
\hline 33 & 45 & $\mathrm{C}$ \\
\hline 13 & 18 & $\mathrm{~A}$ \\
\hline $\mathbf{M = 3 1 , 2}$ & $\mathbf{M}=\mathbf{4 2 , 9}$ & \\
\hline
\end{tabular}

Tabelle 1: Testergebnis der Teilnehmergruppe $(N=10)$ bei der Kreativitätsmessung mit TSD-Z $(A)^{4}$

Aus den einzelnen Punktwerten ergibt sich der TSD-Z-Gesamtwert (siehe Spalte 1: Testwerte), der zu einer groben Einschätzung des kreativen Potenzials dient. Wird der Gesamtwert mit Werten der Normtabelle für Erwachsene (Studierende/Lehrer*innen/Pädagogen*innen) verglichen ${ }^{5}$, kann festgestellt werden, dass die Ergebnisse der Studierenden den Mittelwert der im Manual angegebenen Testsumme (M=31) widerspiegeln. Anhand des Klassifikationsschemas nach Urban und Jelen (1995) können fünf Personen von den Proband*innen mit einer durchschnittlichen Leistung in Kategorie C eingestuft werden, drei Personen in Kategorie D (= überdurchschnittlich) und die Ergebniswerte zweier Probanden liegen weit unter dem Durchschnitt, wodurch sie der Kategorie A zugeordnet werden. Diese Gruppe verfügt laut dem Manual (Urban, 1995) über die geringsten kreativen Ressourcen und Möglichkeiten und ist dadurch besonders förderbedürftig.

In der nächsten Etappe der gestalterischen Arbeit fertigten die Studierenden eine Zeichnung nach einem diktierten Text an. Dabei wurde festgestellt, dass ein und derselbe Text zu vielfältigen Ergebnissen führt. Diese Methode des „Bilddiktats“ regte zu individuellen kreativen Gestaltungen an. Die eigenen subjektiven Vorstellungen von Begriffen wurden mit der jeweiligen zeichnerischen Kompetenz umgesetzt. Eine weitere Aufgabe wurde durch eine Bilderbuchrezeption eingeleitet. Das Bilderbuch von Mies van Hout „Heute bin ich..." zeigt durch expressive Darstellungen von Fischen emotionale Gefühlslagen, die mit einem treffenden Adjektiv schrifttypografisch benannt werden. Durch diese Bild- und Wortimpulse wurden die Studierenden motiviert, ein Nomen oder ein Adjektiv darzustellen. Begriffe wie Freiheit, Glück, Alltag, Corona wurden zur Auswahl angeboten. Des Weiteren sollten die Teilnehmer*innen einen kurzen Text in „Elfchenform" zu einem Bild kreieren. Eine weitere Kreativaufgabe stellte das Erfinden von Bildtiteln dar. Zur Werkanalyse gab es noch Portfolioaufgaben zur Auswahl.

Im zweiten Teil des Workshops näherte sich die Gruppe durch einen Gesprächsimpuls dem Thema „Esskultur“ an. Was befindet sich auf einem feierlich gedeckten Tisch? Im Gespräch entstanden Vorstellungen von unterschiedlichen Tischarrangements. Auf diese Art und Weise erfolgte eine Hinführung zum weltbekannten Kunstwerk „Saliera“ von Benvenutto Cellini, das sich nach einem abenteuerlichen Diebstahl im Jahre 2003 wieder wohlbehalten im KHM in Wien befindet. Die Studierenden wurden über das Kunstwerk informiert und erfuhren ebenso Details über den Kunstraub. Durch all diese Informationen wurde deutlich, welche Wertigkeit Narration im Kontext von Kunstbetrachtungen hat. Die Vorstellungskraft und die Fantasie werden angereichert und erweitert. Eine Weiterführung stellte die Hinwendung zur Textsorte Märchen dar. Die Thematik Salz wurde im Märchen der Salzprinzessin besprochen und der Schlüsselsatz "Ich liebe dich wie..." aufgegriffen und für neue Varianten geöffnet. Die Studierenden wurden eingeladen, ihre persönliche Version dieses Märchens zu erfinden. Abschließend wurde die österreichische Künstlerpersönlichkeit Arnulf Rainer vorgestellt. Seine Übermalungen sind in unterschiedlichen Ausstellungsformaten im Arnulf Rainer Museum in Baden bei Wien zu betrachten. Die Studierenden wurden eingeladen, dieses Museum auf digitalem Weg zu erkunden und sich auf diese Weise ein Bild zu machen. ${ }^{6}$ Somit wurden ein digitaler Museumsbesuch und damit Einblicke in museumsdidaktische Konzepte ermöglicht.

\section{Fazit}

Im Rahmen der abschließenden Besprechung der Portfolioaufgaben wurde durch die Vielfalt der Ergebnisse allen klar, wie unterschiedlich individuell kreative Prozesse ablaufen und welche Heterogenität sich in den Produkten manifestiert. Es war spürbar, mit welchem Interesse der Austausch bei den Präsentationen stattfand und welche Reflexionsprozesse damit ausgelöst wurden. Die Lust auf mehr war entfacht und damit die Wahrscheinlichkeit, dass die Studierenden all diese Methoden ebenso in ihren Unterricht integrieren werden. 


\section{Literatur}

Arn, Ch. (2020). Agile Hochschuldidaktik. Weinheim Basel: Beltz Juvena.

Aljughaiman, A. \& Mowrer-Reynolds, E. (2005). Teachers' conceptions of creativity and creative students. The Journal of Creative Behavior, 39 (Heft 1), S. 17-34.

Beauregard, C. (2014). Effects of classroom-based creative expression programmes on children's well-being. The Arts in Psychotherapy. 41, S. 269-277.

Beghetto, R. A. (2013). Nurturing Creativity in the Micro-moments of the Classroom. In K. H. Kim, J. C. Kaufman, J. Baer, B. Sriraman (Hrsg.), Creatively Gifted Students are not like Other Gifted Students. Advances in Creativity and Giftedness, 5. SensePublishers, Rotterdam (S. 3-15).

Beghetto, R. A., \& Kaufman, J. C. (2014). Classroom contexts for creativity. High Ability Studies, 25 (Heft 1), S. 53-69.

Bereczki, E. O. (2016). Kreativitás és köznevelés: A Nemzeti alaptanterv kreativitásfelfogásának kritikai vizsgálata. Neveléstudomány, 4 (Heft 3), S. 5-20.

Bereczki E. O. \& Kárpáti A. (2018). Teachers' beliefs about creativity and its nurture: a systematic review of the recent research literature. Educational Research Review, 23, S. 25-56.

Berner, N.E. (2013). Bildnerische Kreativität im Grundschulalter; Plastische Arbeiten empirisch betrachtet. München: kopaed.

Berner, N. (Hrsg.) (2018). Kreativität im kunstpädagogischen Diskurs. München: kopaed.

Berner, N., Hess, M. \& Lipowsky F. (2018). Schaffen Freiräume Kreativität? - Offenheit von Gestaltungsaufgaben im Fach Kunst der Grundschule und ihr Potenzial für die Förderung von Kreativität. In E. Glaser, H.C. Koller, W. Thole \& S. Krumme (Hrsg.), Räume für Bildung - Räume der Bildung. Beiträge zum 25. Kongress der Deutschen Gesellschaft für Erziehungswissenschaft. Leverkusen: Verlag Barbara Budrich (S. 259-273).

Brügel, E. (1987). Kunstpädagogische Anmerkungen zu den Komponenten des kreativen Verhaltens. In H. G. Schütz, W. Schiementz (Hrsg.), Kunstpädagogische Einsichten. Beiträge zur Didaktik der Kunst und der ästhetischen Erziehung, Baltmannsweiler: Pädagogischer Verlag, Burgbücherei Schneider (S. 18-41).

Buschkühle, C.-P. (2017). Künstlerische Bildung. Theorie und Praxis einer künstlerischen Kunstpädagogik. Oberhausen: Athena.

Chin, C. (2013). Cultivating divergent thinking: Conceptialization as a critical component of artmaking. Art Education, 66 (Heft 6), 28-32.

Dinkelmann, K. (2008). Kreativitätsförderung im Kunstunterricht. München: Utz.

Einsiedler, W. (1979). Entdeckungslernen im Grundschulunterricht. In W. Einsiedler (Hrsg.), Konzeptionen des Grundschulunterrichts. Bad Heilbrunn/Obb.: Klinkhardt (S. 147-166).

Fadel, C., Bialik, M. \& Trilling, B. (2017). Die vier Dimensionen der Bildung Was Schülerinnen und Schüler im 21. Jahrhundert lernen müssen. Verlag ZLL21, Hamburg.

Fisher, R. \& Williams, M. (2004, Hrsg.). Unlocking Creativity: Teaching Across the Curriculum. Routledge, New York.

Grigorenko, E. L. (2019). Creativity: a challenge for contemporary education. Comparative Education, 55. (Heft 1), S. 116-132.

Harecker, G. (2000). Werterziehung in der Schule: Wege zur Sinnfindung im Unterricht. Facultas Universitätsverlag.

Heinelt, G. (1978). Kreative Lehrer, kreative Schüler. Wie die Schule Kreativität fördern kann. Freiburg im Breisgau: Herder.

Kämpf Jansen, H. (2012). Ästhetische Forschung. Wege durch Alltag, Kunst und Wissenschaft. Zu einem innovativen Konzept ästhetischer Bildung. Marburg: Tectum Verlag.

Kirchner, C. (Hrsg.) (2013). Kunst-Didaktik für die Grundschule. Berlin: Cornelsen.

Kirchner, C. \& Peez, G. (2009). Kreativität in der Grundschule erfolgreich fördern. Arbeitsblätter, Übungen, Unterrichtseinheiten und empirische Untersuchungsergebnisse. Braunschweig: Westermann.

Krautz, J. (2005). Erleben - Machen - Verstehen. Praktische Kunstrezeption zwischen Kunst und Subjekt. BÖKWE - Fachblatt des Bundes Österreichischer Kunst- und Werkerzieher (2), S. 15-22.

Lai, E. R., Yabro, J., DiCerbo, K. \& DeGeest, E. (2018). Skills for today: What we know about teaching and assessing creativity. Pearson, London. Psychologist, 39 (Heft 2), S. 83-96.

Lehrplan der Volksschule, https://www.bmbwf.gv.at/Themen/schule/schulpraxis/lp/lp vs.html, als pdf abgerufen am 20.07.2021 
Ma, H. H. (2006). A synthetic analysis of the effectiveness of single components and packages in creativity training programs. Creativity Research Journal, 18, S. 435-446.

Moran, S. (2010). Creativity in school. In K. Littleton, C. P. Wood, J. Kleine Staarman (Hrsg.), International handbook of psychology in education, England: Emerald, S. 319-359.

Morgan, S. \& Forster, J. (1999). Creativity in the classroom. Gifted Education International, 14, S. $29-43$.

Otto, G. \& Otto, M. (1987). Auslegen. Ästhetische Erziehung als Praxis des Auslegens in Bildern und des Auslegens von Bildern. Seelze: Friedrich.

Plucker, J., Beghetto, R. \& Dow, G. (2004). Why isn't creativity more important to Educational Psychologists? Potentials, pitfalls, and future directions in creativity research. Educational Psychologist, 39 (Heft 2), S. 83-96.

Preiser, S. \& Buchholz, N. (2008). Kreativität. Kröning: Asanger Verlag.

Rosa, H. (2017). Resonanz. Eine Soziologie der Weltbeziehung. Berlin: Suhrkamp. S. 472-500.

Sawyer, K. (2012). Explaining creativity: The science of human innovation (Bd. 2). New York: Oxford University Press.

Seitz, R. (2009). Kreative Kinder. Das Praxisbuch für Eltern und Pädagogen. München: Kösel.

Scott, C. (1999). Teachers' biases toward creative children. Creativity Research Journal, 12 (Heft 4), S. 321-328.

Scott, G., Leritz, L. E. \& Mumford, M. D. (2004a). The effectiveness of creativity training: A quantitative review. Creativity Research Journal, 16., S. 361-388.

Scott, G. M., Leritz, L. E. \& Mumford, M. D. (2004b). Types of creativity training: aproaches and their effectiveness. The Journal of Creative Behavior, 38 (Heft 3), S. 149-179.

Schäfer, L. (2006). Der Zirkel des Schaffens. Neue Deutungen von Kreativität und ihre Relevanz für den Kunstunterricht. Oberhausen: Athena.

Schuster, M. (1997). Künstlerische Kreativität. Der Versuch einer kreativen Auseinandersetzung. In H. R. Becher, M. Schuster \& C. Mauer (Hrsg.), Taschenbuch: Kunst, Pädagogik, Psychologie. Kunststandpunkte Baltmannweiler: Schneider-Vlg. Hohengehren. (S. 66-80).

„SCHÜLER\&INNEN_KOMPETENZEN_BE LEITFADEN10|2013“. Wien. BMUKK.

Theurer, Caroline (2015). Kreativitätsförderndes Klassenklima als Determinante der Kreativitätsentwicklung von Grundschulkindern. KREAplus (Bd. 7), kopaed, München.

Urban, K. K.: (2011). Möglichkeiten und Grenzen von Kreativitätsdiagnostik. In C. Koop, O. Steenbuck (Hrsg.), Kreativität: Zufall oder harte Arbeit? Karg-Hefte. Beiträge zur Begabtenförderung und Begabungsforschung (Bd. 2), Frankfurt, M.: Karg-Stiftung (S. 18-27).

Urban, K. K. (2004). Kreativität. Herausforderung für Schule, Wissenschaft und Gesellschaft. Münster: LIT Verlag.

Urban, K. K. \& Jellen, H. G. (1995). Test zum schöpferischen Denken - Zeichnerisch (TSD-Z). Frankfurt am Main: Swets.

Ursprung, P. (2012). Die Kunst der Gegenwart. 1960 bis heute. München: Beck.

Van Hout, M. (2012). Heute bin ich. Zürich: aracariverlag.

Westby, E. \& Dawson, V. (1995). Creativity: Asset or burden in the classroom? Creativity Research Journal, 8 (Heft 1), S. 1-10.

Wichelhaus, B. (2006). Fördern im Kunstunterricht. Prinzipien, Perspektiven und Probleme. Kunst + Unterricht, 307-308, S. 3-10.

\footnotetext{
125 Anregungen zur Kreativitätsförderung von Klaus K. Urban https://besondersbegabte.alp.dillingen.de/images/Dokumente_red/Unterricht/25_anregungen_zur_kreativitaetsfoerderun g.pdf (28.07.2021)

2 https://www.bmbwf.gv.at/Themen/schule/schulpraxis/lp/lp vs.html (20.07.2021)

${ }^{3}$ siehe „SCHÜLER\&INNEN_KOMPETENZEN_BE LEITFADEN10|2013, Wien. BMUKK, S. 14.

${ }^{4}$ Testblätter erhielten von etwa der Hälfte der Teilnehmer.

${ }^{5}$ vgl. Urban. Manual TSD-Z, 1995, S.41

${ }^{6}$ https://www.arnulf-rainer-museum.at/
} 